\title{
The effect of team collaboration and continuity of care on health and disability among rehabilitation patients: a longitudinal survey-based study from western Norway
}

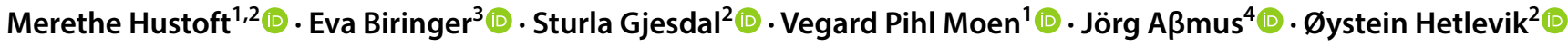

Accepted: 23 May 2019 / Published online: 29 May 2019

(C) The Author(s) 2019

\begin{abstract}
Purpose The purpose of this study was to investigate how changes in patient-rated health and disability from baseline to after rehabilitation were associated with communication and relationships in rehabilitation teams and patient-rated continuity of care.

Methods Linear models were used to assess the associations between relational coordination [RC] and Nijmegen Continuity Questionnaire-Norwegian version [NCQ-N] with changes in the World Health Association Disability Assessment Schedule 2.0 [WHODAS 2.0] and EuroQol EQ-VAS [EQ-VAS]. To express change in WHODAS 2.0 and EQ-VAS, the model was adjusted for WHODAS 2.0 and EQ-VAS baseline scores. Analyses for possible slopes for the various diagnosis groups were performed.

Results A sample of 701 patients were included in the patient cohort, followed from before rehabilitation to 1 year after a rehabilitation stay involving treatment by 15 different interprofessional teams. The analyses revealed associations between continuity of care and changes in patient-rated health, measured with EQ-VAS (all $p$ values $<0.01$ ). RC communication was associated with more improvement in functioning in neoplasms patient group, compared to improvement of health among included patient groups. The results revealed no associations between NCQ-N and WHODAS 2.0 global score, or between $\mathrm{RC}$ in the rehabilitation teams treating the patients and changes in WHODAS 2.0 global score.

Conclusion The current results revealed that better personal, team and cross-boundary continuity of rehabilitation care was associated with better patient health after rehabilitation at 1-year follow-up. Measures of patient experiences with different types of continuity of care may provide a promising indicator of the quality of rehabilitation care.
\end{abstract}

Keywords Continuity of patient care $\cdot$ Rehabilitation $\cdot$ Disability evaluation $\cdot$ Interprofessional relations $\cdot$ Patient-rated outcome measures $\cdot$ Relational coordination

Electronic supplementary material The online version of this article (https://doi.org/10.1007/s11136-019-02216-7) contains supplementary material, which is available to authorized users.

Merethe Hustoft

merethe.hustoft@helse-bergen.no

1 Centre for Habilitation and Rehabilitation in Western Norway, Haukeland University Hospital, Bergen, Norway

2 Department of Global Health and Primary Health Care, University of Bergen, Bergen, Norway

3 Section of Research and Innovation, Helse Fonna Local Health Authority, Haugesund/Stord, Norway

4 Centre for Clinical Research, Haukeland University Hospital, Bergen, Norway

\begin{tabular}{|c|c|}
\hline \multicolumn{2}{|l|}{ Abbreviations } \\
\hline $\mathrm{RC}$ & Relational coordination \\
\hline NCQ & Nijmegen Continuity Questionnaire \\
\hline NCQ-N & $\begin{array}{l}\text { Norwegian version of the Nijmegen Con- } \\
\text { tinuity Questionnaire }\end{array}$ \\
\hline GP & General practitioner \\
\hline WHODAS 2.0 & $\begin{array}{l}\text { World Health Organization Disability } \\
\text { Assessment Schedule } 2.0\end{array}$ \\
\hline EQ-5D-5L & EuroQol-5 dimension descriptive system \\
\hline EQ-VAS & EuroQol EQ-VAS \\
\hline $\mathrm{b}$ & $\begin{array}{l}\text { Unstandardized estimated regression } \\
\text { coefficient }\end{array}$ \\
\hline SD & Standard deviation \\
\hline CI & Confidence interval \\
\hline
\end{tabular}




\section{Background}

Rehabilitation is considered one of the most important processes enabling attainment and maintenance of physical, mental, social and vocational activities for people with various health conditions and disabilities [1]. Somatic rehabilitation emphasises health and functioning through a continuous and coordinated process that extends over a period of time with a collaborating interprofessional rehabilitation team [2]. Self-rated health and disability have received increased attention in recent decades as important outcomes in rehabilitation [3].

While undergoing rehabilitation, patients are treated by an array of health care professionals in a team, not only during their stay in a rehabilitation centre, but also across multiple specialities and in different health care settings [4]. According to Donabedian's health care quality model, high-quality structures of care should lead to improvements in clinical processes and subsequently improve patient outcomes [5]. Collaboration and coordination in interprofessional rehabilitation teams are important for ensuring good quality continuity of care and outcomes for patients [6-8]. Relational coordination (RC) among interprofessional team members has been found to improve patient outcomes [9] and impact care coordination [10].

Continuity of rehabilitation care occurs when patient experiences are linked to care over time or when the care is connected [11]. Continuity of care is considered to be essential for high-quality patient care [12-14] and is commonly framed as being composed of relational continuity (relationship between a patient and a provider over time), information continuity (availability and use of data from prior events during current patient encounters) and management continuity (coherent delivery of care from different health care professionals) $[11,13]$. It is generally preferable for continuity of care to be measured from the patients' perspective [15].

A large number of studies of continuity of care have examined the personal continuity between patients and general practitioner (GP) or health care professional delivering care over time and have typically been performed in primary health care settings $[16,17]$. Few studies have investigated continuity of care in somatic specialised health care and even fewer have examined somatic rehabilitation settings [18-20]. Investigations of patients' perceived personal, team and cross-boundary continuity in rehabilitation services are scarce [21]. In a recent study, we found associations between $\mathrm{RC}$ functions in interprofessional rehabilitation teams and the patient-rated continuity of care at 1-year follow-up [18]. Further, this previous study also indicated weak associations between RC subscale scores and patient-rated benefit in more general terms, most pronounced related to activities in daily living [18].
To the best of our knowledge, no previous studies have investigated the associations between interprofessional team functioning and continuity of care with changes in patient-rated health and disability longitudinally. Therefore, we assessed associations between RC in interprofessional rehabilitation teams and patient-rated continuity of care with changes in patient-rated health and disability.

\section{Aims}

The current study sought to investigate how changes in patient-rated health and disability from baseline to after rehabilitation were associated with communication and relationships in rehabilitation teams and patient-rated continuity of care.

\section{Methods}

\section{Study design}

This study used a longitudinal survey-based design following a cohort of patients accepted for a rehabilitation stay in secondary health care services (Fig. 1). Survey data was collected when patients were recruited (baseline) and in a follow-up survey 1 year after baseline data collection. In between the two surveys, the patients had a rehabilitation stay in one of the centres. All patients included have taken part in a 3-week rehabilitation process treated by an interprofessional team comprising of a physician, occupational therapist, physical therapist, nurse and other relevant team members. Each of the seven rehabilitation centres in Western Norway provides interventions appropriate for the diagnostic group referred to the centre. As we aimed to include a large cohort of rehabilitation patients in Western Norway all patients who were referred with various diagnosis were included, and therefore a single specific intervention is not studied. RC in interprofessional teams were estimated by a survey among the professionals working in the rehabilitation centres.

\section{Participants}

\section{Patient cohort}

All patients aged 18 and above who were accepted for rehabilitation in a rehabilitation centre in Western Norway between January 2015 and June 2015 were invited to participate $(n=2863)$. For baseline data collection, a total of $984(34 \%)$ patients accepted the invitation to participate and provided written consent and a completed questionnaire. A 1-year follow-up questionnaire was sent to all participating patients $(n=984)$, and 705 patients $(25 \%$ of the 
Fig. 1 Flow chart for data collection

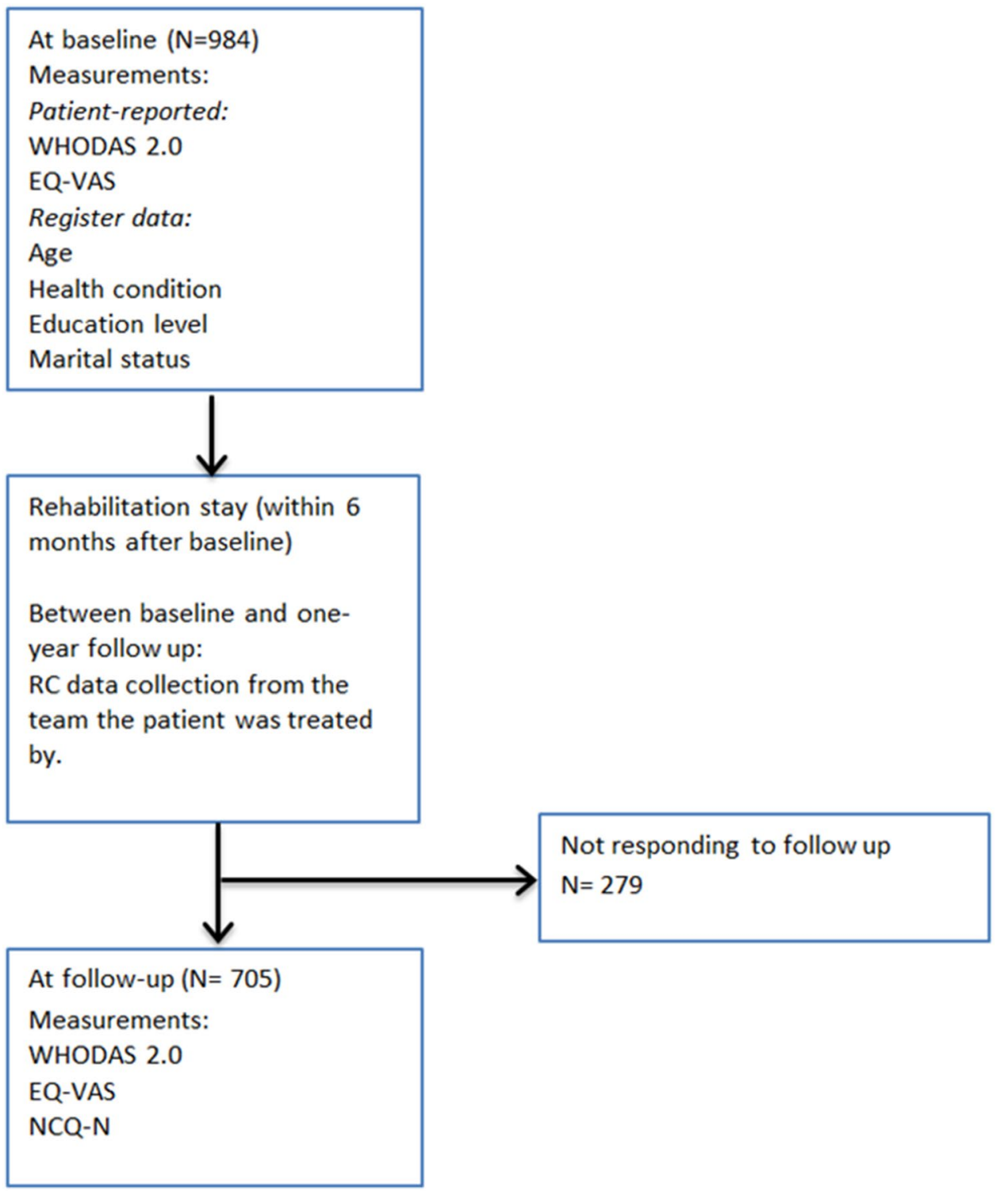

patient group invited at baseline) returned the questionnaire. We extracted 279 of the baseline participants from the analyses, as they did not respond to the 1-year followup survey. Four respondents were omitted from the analyses due to missing data on outcome variables. Finally, 701 ( $24 \%$ of the patient group invited at baseline) patients were included in the analyses (Table 1). Each patient respondent was linked to their corresponding interprofessional team from whom they received rehabilitation services during their stay in the rehabilitation centre. Further descriptions of the recruitment and inclusion process of patients and health care professionals have been reported in previous studies [18, 22, 23].

\section{Dependent variables and measurements}

The World Health Organization Disability Assessment Schedule version 2.0 (WHODAS 2.0) was developed to correspond directly to the "activity and participation" dimension of the International Classification of Disability, Function and Health (ICF) [24] and has previously been used to evaluate disability in a generic rehabilitation group $[25,26]$. WHODAS 2.0 is an extensively validated and used patient-rated generic self-evaluation survey instrument [22, 27, 28]. WHODAS 2.0 is translated into several languages, including Norwegian [22], and has been used 
Table 1 Characteristics of included rehabilitation patients $(N=701)$ answering both baseline and the 1-year follow-up survey, and nonresponders of the 1-year follow-up survey $(N=279)$

\begin{tabular}{|c|c|c|}
\hline Patient characteristics & $\begin{array}{l}\text { Included patients } \\
(N=701)\end{array}$ & $\begin{array}{l}\text { Non-responders at } \\
1 \text {-year follow-up } \\
(N=279)\end{array}$ \\
\hline \multicolumn{3}{|l|}{ Age mean (SD) } \\
\hline Male & $63(13.4)$ & $56(12.83)$ \\
\hline Female & $60(13.5)$ & $52(15.12)$ \\
\hline \multicolumn{3}{|l|}{ Age group $n(\%)$} \\
\hline $18-29$ & $10(1.4)$ & $12(4.6)$ \\
\hline $30-39$ & $35(5.0)$ & $40(14.5)$ \\
\hline $40-49$ & $113(16.1)$ & $68(24.5)$ \\
\hline $50-59$ & $165(23.5)$ & $65(23.5)$ \\
\hline $60-69$ & $198(28.3)$ & $52(18.8)$ \\
\hline$>70$ & $180(25.7)$ & $39(14.1)$ \\
\hline Missing & $0(0)$ & $0(0)$ \\
\hline \multicolumn{3}{|l|}{$\operatorname{Sex} n(\%)$} \\
\hline Male & $269(38.0)$ & $88(31.5)$ \\
\hline Female & $432(62.0)$ & $191(68.5)$ \\
\hline Missing & $0(0)$ & $0(0)$ \\
\hline \multicolumn{3}{|l|}{ Health conditions $n(\%)$} \\
\hline Neoplasms & $49(7.0)$ & $16(5.7)$ \\
\hline Diseases in the nervous system & $81(11.6)$ & $21(7.5)$ \\
\hline Diseases in the musculoskeletal system & $356(50.8)$ & $130(46.6)$ \\
\hline Diseases in the circulatory system & $60(8.6)$ & $48(17.2)$ \\
\hline Others $^{\mathrm{a}}$ & $152(21.7)$ & $64(23.0)$ \\
\hline Missing & $3(0.4)$ & $0(0)$ \\
\hline \multicolumn{3}{|l|}{ Education level $n(\%)$} \\
\hline Elementary school & $152(21.7)$ & $76(27.2)$ \\
\hline High school & $328(46.8)$ & $128(45.9)$ \\
\hline College/University & $213(30.4)$ & $67(24.0)$ \\
\hline Missing & $8(1.1)$ & $8(2.9)$ \\
\hline \multicolumn{3}{|l|}{ Marital status $n(\%)$} \\
\hline Married & $356(50.8)$ & $130(46.6)$ \\
\hline Unmarried, not divorced & $189(27.0)$ & $83(29.7)$ \\
\hline Divorced & $150(21.4)$ & $64(22.9)$ \\
\hline Missing & $6(0.9)$ & $2(0.7)$ \\
\hline
\end{tabular}

${ }^{a}$ Other health conditions included the following: endocrine, nutritional and metabolic diseases $(n=36)$; respiratory diseases $(n=35)$; diseases of the skin and subcutaneous tissue (23); injuries and external causes $(n=18)$; factors influencing self-rated health and contact with services $(n=7)$; mental and behavioural disorders $(n=12)$; symptoms, sign and abnormal clinical and laboratory findings, not elsewhere classified $(n=4)$; codes for special purposes $(n=6)$; diseases of the digestive system $(n=5)$; diseases of the blood and blood-forming organs, and certain disorders involving the immune mechanism $(n=1)$; diseases of the ear and the mastoid process $(n=1)$; diseases of the genitourinary system $(n=1)$; congenital malfunctions, and chromosomal abnormalities $(n=1)$; and certain infectious and parasitic diseases $(n=2)$ in various health care settings, such as chronic care [24], stroke [29] and secondary rehabilitation services [23].

WHODAS 2.0 measures health and disability using 36 items across six domains [26] (number of items and Cronbach's alpha from Norwegian validation study [22] in parentheses): cognition ( $\alpha=0.85$ ), self-care (four items, $\alpha=0.77$ ), getting along (five items, $\alpha=0.75$ ), life activities (eight items, $\alpha=0.91$ ) and participation (eight items, $\alpha=0.83$ ). Four items in the domain of life activities relate to the household and four items relate to work/study. Responses were given on a fivepoint Likert scale $($ one $=$ none, two $=$ mild, three $=$ moderate, four $=$ severe and five $=$ extreme or cannot do). Scores were computed for each domain by adding the item responses representing each domain. Each domain score was transformed into a range from zero (best $=$ no disability) to 100 (worst $=$ full disability). A global score was calculated using either all 36 items or 32 items in cases where the four items 
regarding work/school were omitted because they did not apply to the participating patients [30]. The global score ranged from zero (best $=$ no disability) to 100 (worst $=$ full disability). The range scores for the domain and global scores were assessed as 0-4: no functional problem; 5-24: mild functional problem; 25-40: moderate functional problem; 50-95: severe functional problem and 95-100: total functional loss. The calculation of the WHODAS 2.0 domain and global scores was conducted according to the WHODAS 2.0 manual with complex scoring [26].

The EuroQol-5 dimension descriptive system (EQ-5D) includes a visual analogue scale (EQ-VAS) for measuring respondents' overall health status [31-34]. The EQ-5D is an extensively validated and reliable generic health-related measurement tool [35-37], including validation in rehabilitation settings [31, 32]. EQ-5D has, among others, been used in primary care [38], geriatric health [39] and in somatic and community-based rehabilitation settings [23, 40]. EQ-5D is ideally used by self-evaluation [34]. Respondents indicated their self-rated health on a vertical, calibrated, line ranging from zero ("worst imaginable health state") to 100 ("best imaginable health state") [34].

\section{Independent variables and measurements}

The main independent variables in this study were the teamreported RC subscale scores and the patient-rated Nijmegen Continuity Questionnaire, Norwegian version, (NCQ-N) subscale scores.

$\mathrm{RC}$ is a self-reporting validated survey measuring team functions among members of interprofessional teams [20, 41]. The RC survey has recently been translated into Norwegian and validated within teams in specialised health care settings [42]. This study found a satisfactory two-factor solution (Cronbach's alpha in parentheses); RC communication $=$ four items: frequency, accuracy, timeliness and problem-solving $(\alpha=0.93), \mathrm{RC}$ relationship $=$ three items: shared knowledge, shared goals, mutual respect) $(\alpha=0.80)[42,43]$. Each item represents a question (e.g. "How frequently do members of the interprofessional team communicate with you about the rehabilitation patient?"). Responses were reported on a 5-point Likert scale $($ one $=$ never, two $=$ rarely, three $=$ occasionally, four $=$ often and five $=$ always $). R C$ has been used in various health care settings, such as primary health [44], hospital settings [42, 45] and secondary rehabilitation services [18]. RC subscale scores were obtained for all teams $(N=15)$ in all rehabilitation centres by conducting a survey among health care professionals $(N=124$, $52 \%$ response rate). The $\mathrm{RC}$ subscale scores are reported as clustered mean scores for each team in this study, and scores were assigned to the patients treated by the respective teams.

The Nijmegen Continuity Questionnaire (NCQ) is a validated generic survey measuring continuity of care from the perspectives of the patients and consists of 28 items divided into six subscales [46, 47]. The NCQ has been used in primary care [19], chronic illness [48] and somatic rehabilitation [18, 49]. The NCQ has recently been translated into Norwegian (NCQ-N) [49]. In this study, we used two subscales of the NCQ-N for personal continuity (number of items and Cronbach's alpha in parentheses): most important health care professional in the interprofessional rehabilitation team knows me (five items, $\alpha=0.92$ ), most important health care professional in the interprofessional rehabilitation team shows commitment (three items, $\alpha=0.88$ ) together with subscales regarding team continuity: collaboration between providers within the team in the rehabilitation centre (four items, $\alpha=0.96$ ) and cross-boundary continuity: between the rehabilitation centres and general practitioners in the municipality (four items, $\alpha=0.95$ ). The NCQ-N uses a 5 -point Likert scale $($ one $=$ strongly disagree, two $=$ disagree, three $=$ neutral, four $=$ agree, five $=$ strongly agree) with an option of "don't know" (set as missing).

As adjustment variables we used variables; age and sex from the baseline survey. Variables; marital status and $e d u$ cation level were register data provided by Statistics Norway and linked to the survey.

\section{Statistical analyses}

Descriptive methods were used to describe sample characteristics. Missing data was handled with flexible multiple imputation method using chained predictive mean matching, creating 50 datasets [50]. Rubin's rules were used for pooling the results [50].

Linear models were used to assess the association between RC and NCQ-N as independent variables and the WHODAS 2.0 domain and global scores and EQ-VAS score at 1-year as dependent variables. To express change in WHODAS 2.0 and EQ-VAS from baseline to follow-up, the model was also adjusted for WHODAS 2.0 and EQ-VAS baseline scores [51]. All models were adjusted for: sex, age (categorised as: $18-29,30-39,40-49,50-59,60-69$ and $>70$ ), marital status(categorised as: married, unmarried [not divorced], divorced), education level (categorised as: elementary school, high school and university/college) and health conditions, based on the Statistical Classification of Diseases and Related Health Problems Tenth Revision (ICD10) referral diagnosis grouped as: neoplasms, nervous system diseases, musculoskeletal system diseases, circulatory system diseases, and others. Additionally, we made corresponding analyses including an interaction between diagnoses and the independent variables to assess possibly different slopes for the various ICD-10 referral diagnosis groups. All $\mathrm{RC}$ scales at patient level were clustered because of the team allocation. This has been taken into account by adding a 
random intercept for team allocation in the models including $\mathrm{RC}$, turning them to Linear Mixed Effects models (LME).

The level of significance was set as 0.05 . All statistical analyses were performed with IBM SPSS for Windows version 24 (IBM Corp. Armonk, NY) [52], and STATA 15 (STATA Corp., College Station, TX) [53]. The graphics were produced using Matlab 9.0 (The Mathworks Inc., Natrick, MA).

\section{Results}

Patients reported a mean WHODAS 2.0 global score at 28.6 (standard deviation $[\mathrm{SD}]=15.4$ ) at baseline, which decreased to $24.1(\mathrm{SD}=15.9)$ at 1 -year follow-up, indicating reduced disability. Patients with neoplasms reported a larger reduction of disability, as measured by WHODAS 2.0 global score, compared to patients in other referral diagnosis groups included in this study (Table 2). The mean EQ-VAS score changed from $51.4(\mathrm{SD}=18.8)$ at baseline to 58.2 $(\mathrm{SD}=20.1)$ at 1 -year follow-up, indicating improved selfrated health. Generally, patients reported largest reduction of disability for the WHODAS 2.0 domains: life activities, mobility and participation domains (Table 2). The neoplasms patient group shows a market reduction of disability in most WHODAS 2.0 domain scores and EQ-VAS score compared to other referral diagnosis groups included in this study (Supplementary Table 1).

The mean interprofessional team RC communication score for the patient group was $3.9(\mathrm{SD}=0.31)$, and the mean team $\mathrm{RC}$ relationship score for the patient group was 4.1 $(\mathrm{SD}=0.28)$ (Table 3). NCQ-N among patients ranged from $2.9(\mathrm{SD}=0.91)$ for personal continuity, where respondents

Table 3 Relational coordination and Nijmegen Continuity Questionnaire-N subscale scores in the study population $(N=701)$

\begin{tabular}{ll}
\hline & Mean (SD) \\
\hline Relational coordination a & \\
RC communication & $3.9(0.31)$ \\
RC relationship & $4.1(0.28)$ \\
Nijmegen Continuity Questionnaire-Norwegian version & \\
NCQ-N personal continuity ("knows me") & $3.0(0.83)$ \\
NCQ-N personal continuity ("shows commitment") & $2.9(0.91)$ \\
NCQ-N team continuity (within somatic rehabilitation) & $3.7(0.84)$ \\
NCQ-N cross-boundary continuity (between rehabilita- & $3.0(0.92)$ \\
tion centres and GP in municipality) & \\
\hline
\end{tabular}

$R C$ relational coordination, $N C Q-N$ Nijmegen continuity questionnaire-Norwegian version, $G P$ general practitioner, $S D$ standard deviation

${ }^{a}$ All patients were connected to their respective treating team in the rehabilitation centre during their stay
Table 2 Distribution of the World Health Organisation Disability Assessment Schedule 2.0 and the EuroQol EQ-VAS among 701 patients at baseline and 1-year follow-up from specialised rehabilitation centres in Western Norway during the first half of 2015 and 2016

\begin{tabular}{llll}
\hline & $\begin{array}{l}\text { Baseline } \\
\text { Mean (SD) }\end{array}$ & $\begin{array}{l}\text { 1-year follow-up } \\
\text { Mean (SD) }\end{array}$ & $\begin{array}{l}\text { Change score } \\
\text { Mean (95\% CI) }\end{array}$ \\
\hline WHODAS 2.0 domain score (all patients) & & & \\
Cognition & $16.4(18.0)$ & $14.3(16.4)$ & $-2.1(-3.24,-0.96)$ \\
Mobility & $32.5(25.4)$ & $26.3(25.2)$ & $-6.2(-7.77,-4.63)$ \\
Self-care & $11.0(17.2)$ & $8.4(15.9)$ & $-2.6(-3.84,-1.36)$ \\
Getting along & $23.9(20.7)$ & $22.3(21.4)$ & $-1.6(-2.93,-0.27)$ \\
Life activities & $43.5(28.1)$ & $34.8(27.5)$ & $-8.7(-10.62,-6.78)$ \\
Participation & $39.4(20.4)$ & $34.6(21.7)$ & $-4.8(-6.10,-3.50)$ \\
WHODAS 2.0 global score (all patients) & $28.6(15.4)$ & $24.1(15.9)$ & $-4.5(-5.42,-3.58)$ \\
Neoplasms & $30.3(15.4)$ & $20.1(14.8)$ & $-10.2(-14.83,-5.57)$ \\
Diseases in nervous systems & $30.0(14.2)$ & $26.4(14.0)$ & $-3.6(-6.08,-1.18)$ \\
Diseases in musculoskeletal systems & $26.6(15.3)$ & $22.2(15.9)$ & $-4.4(-5.57,-3.13)$ \\
Diseases in circulatory systems & $32.6(15.7)$ & $28.4(16.6)$ & $-4.2(-7.39,-1.03)$ \\
Others & $30.6(15.0)$ & $27.1(16.3)$ & $-3.5(-5.48,-1.52)$ \\
EQ-VAS (all patients) & $51.4(18.8)$ & $58.2(20.1)$ & $7.2(5.85,8.55)$ \\
Neoplasms & $51.7(19.7)$ & $63.4(21.9)$ & $10.2(3.17,17.17)$ \\
Diseases in nervous systems & $46.1(18.9)$ & $56.3(18.3)$ & $9.7(5.92,13.52)$ \\
Diseases in musculoskeletal systems & $53.0(18.7)$ & $59.9(19.8)$ & $7.0(5.29,8.77)$ \\
Diseases in circulatory systems & $47.4(17.0)$ & $55.2(16.9)$ & $8.0(3.15,12.83)$ \\
Others & $50.6(19.1)$ & $54.6(21.0)$ & $4.6(1.61,7.53)$ \\
\hline
\end{tabular}

WHODAS 2.0, World Health Organization Disability Assessment Schedule version 2.0; EQ-VAS, EuroQol EQ-VAS; SD: standard deviation; 95\% CI, 95\% confidence interval; 1: WHODAS 2.0 domain and global score range from: $0=$ no disability to $100=$ full disability); 2: EQ-VAS range from, $0=$ worst imaginable health state to $100=$ best imaginable health state 
Table 4 Associations of relational coordination in interprofessional teams and patient-rated continuity of care subscale scores with the changes in World Health Organisation Disability Assessment Schedule 2.0 global score $(N=701)$

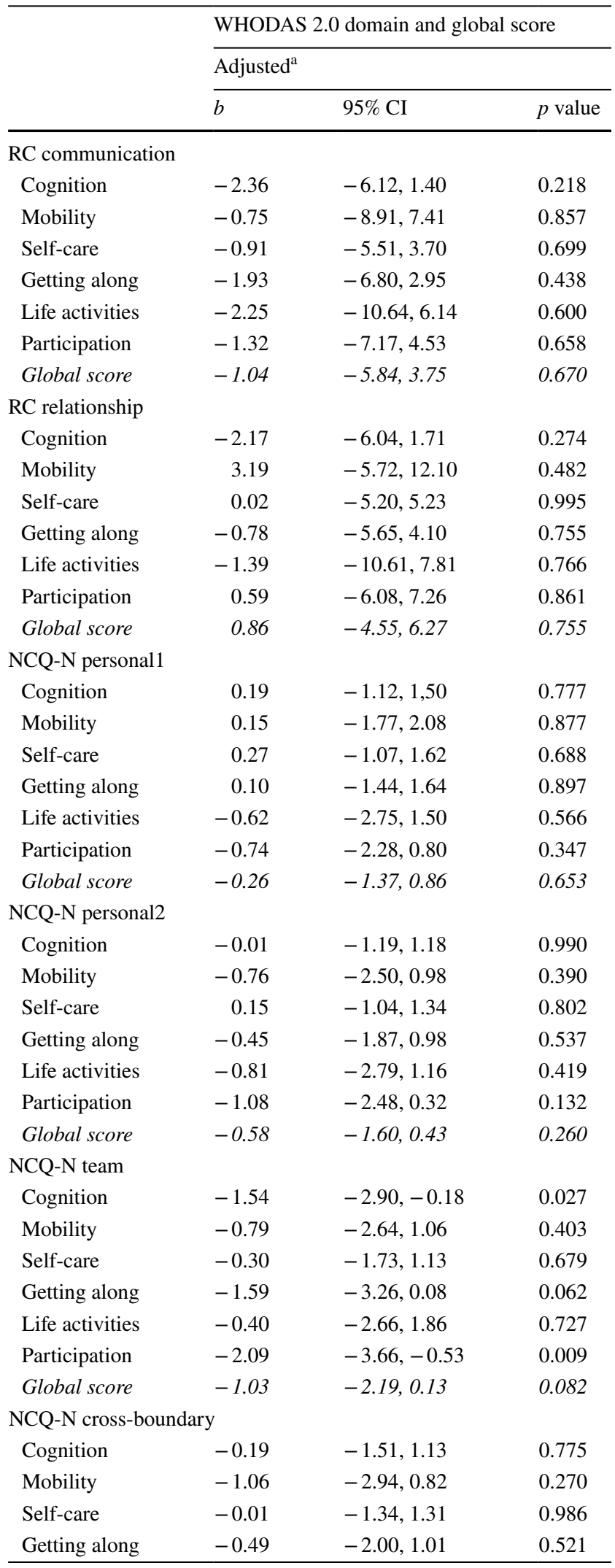

Table 4 (continued)

\begin{tabular}{llll}
\hline & \multicolumn{4}{l}{ WHODAS 2.0 domain and global score } \\
\cline { 2 - 4 } & \multicolumn{2}{l}{ Adjusted $^{\mathrm{a}}$} \\
\cline { 2 - 4 } & $b$ & $95 \%$ CI & $p$ value \\
\hline Life activities & -2.20 & $-4.39,-0.00$ & 0.050 \\
Participation & -1.26 & $-2.84,0.31$ & 0.115 \\
Global score & -0.79 & $-1.97,0.38$ & 0.186 \\
\hline
\end{tabular}

WHODAS 2.0 World Health Organization Disability Assessment Schedule version 2.0, $R C$ relational coordination subscale score, $N C Q-N$ Nijmegen continuity questionnaire- Norwegian version, $b$ unstandardized estimated regression coefficient, $C I$ confidence interval, NCQ-N Personall NCQ-N personal continuity ("knows me"), NCQ-N Personal 2 NCQ-N personal continuity ("shows commitment"), NCQ-N Team NCQ-N team continuity (within somatic rehabilitation), NCQ-N Cross-boundary NCQ-N cross-boundary continuity (between rehabilitation centres and general practitioner in municipality)

aAdjusted for: patients' age group, sex, health conditions, education level, marital status and baseline dependent variable subscale score (WHODAS 2.0)

reported that the most important health care professional in the team "shows commitment", to the highest mean score for team continuity within somatic rehabilitation centres of $3.7(\mathrm{SD}=0.84)($ Table 3$)$.

No associations were found between RC and NCQ-N subscale with changes in WHODAS 2.0 global score (Table 4). There were associations between NCQ-N team continuity and change in WHODAS 2.0 cognition; $-1.54(\mathrm{SD}=18.3$, $p=0.027$ ), NCQ-N team continuity and WHODAS 2.0 participation; -2.09 ( $\mathrm{SD}=21.2, p=0.009)$ and NCQ-N crossboundary continuity and WHODAS 2.0 life activities; -2.20 $(\mathrm{SD}=29.7, p=0.050)$; however, no associations were found between RC and changes in WHODAS 2.0 domain scores (Table 4).

Figure 2 presents analyses of associations between RC and NCQ-N subscale scores and changes in WHODAS 2.0 global scores for patient grouped by referral diagnosis. A higher RC communication score was associated with improved health for the neoplasms patient group $(b=-20.66,95 \% \mathrm{CI}=-37.05,-4.28, p=0.013)$ (Supplementary Table 3). A similar (not significant) pattern can be seen between RC relationship and WHODAS 2.0 global scores for the neoplasms patient group. This study did not disclose associations between NCQ-N and changes in WHODAS 2.0 global score when analysing referral diagnosis groups separately. Supplementary Table 3 provides $b$ coefficient, 95\% CI and $p$ values related to Fig. 2.

We found significant associations between all NCQ-N subscales and changes in the EQ-VAS (Table 5), while no associations were found between $\mathrm{RC}$ and changes in EQ-VAS. 


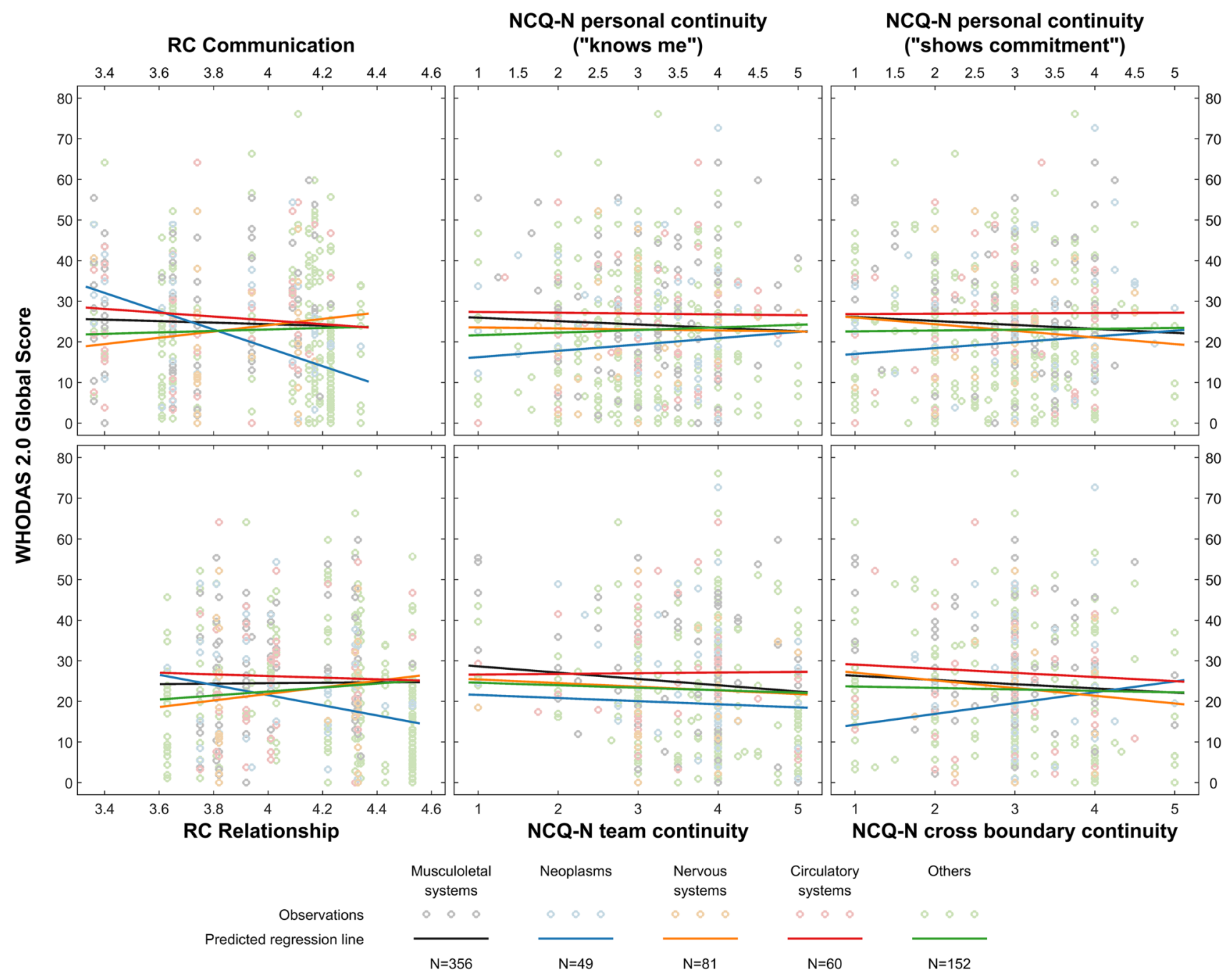

Fig. 2 Associations of relational coordination subscale scores in interprofessional teams and patient-rated continuity of care subscale scores with the World Health Organisation Disability Assessment Schedule 2.0 global score with patients grouped by ICD-10 referral

Figure 3 presents analyses of associations between RC and NCQ-N subscale scores with changes in EQ-VAS scores for patients grouped by referral diagnosis. Patients referred with nervous system diseases reported a decrease in the EQ-VAS score when treated by teams with higher levels of RC relationship score $(b=-20.66,95 \% \mathrm{CI}=-38.96$, $-2.36, p=0.027$ ) (Supplementary Table 4), a similar (not significant) association was seen between RC communication score and EQ-VAS score in the same patient group. This study found that patients in all referral diagnosis groups reported improvement in health when experiencing continuity of care. Supplementary Table 4 provides b coefficient, 95\% CI and $p$ values related to Fig. 3 . diagnoses $(N=701)$. WHODAS 2.0 World Health Organization Disability Assessment Schedule version 2.0, $R C$ relational coordination, $N C Q-N$ Nijmegen continuity questionnaire-Norwegian version

\section{Discussion}

To the best of our knowledge, this is the first study to investigate the associations between team functions in somatic rehabilitation centres and changes in health and disability among rehabilitation patients. An improvement of health was associated with better patient-reported continuity of care regarding rehabilitation care. However, continuity of care was not associated with reduced disability. Communication and relationship in teams, as reported by the professionals, were not associated with improvement in health or decreased disability, looking at the total sample. However, neoplasms patient group improved their health more compared to other diagnosis groups included in this study.

Previous studies have reported that continuity of care is associated with reduced length of stay in hospital, reduced 
Table 5 Associations of relational coordination subscale scores in interprofessional teams and patient-rated continuity of care subscale scores with the EuroQol EQ-VAS health state score $(N=701)$

\begin{tabular}{llll}
\hline & \multicolumn{4}{l}{ EQ-VAS score } \\
\cline { 2 - 4 } & \multicolumn{2}{l}{ Adjusted $^{\mathrm{a}}$} \\
\cline { 2 - 4 } & $b$ & $95 \%$ CI & $p$ value \\
\hline RC communication & 0.99 & $-5.49,7.46$ & 0.764 \\
RC relationship & 0.27 & $-6.90,7.44$ & 0.941 \\
NCQ-N Personal1 & 2.50 & $0.94,4.06$ & 0.002 \\
NCQ-N Personal2 & 2.28 & $0.81,3.76$ & 0.002 \\
NCQ-N team & 1.73 & $0.11,3.35$ & 0.037 \\
NCQ-N cross-boundary & 2.40 & $0.84,3.96$ & 0.003 \\
\hline
\end{tabular}

$E Q$-VAS EuroQol EQ-VAS, $R C$ relational coordination subscale score, $N C Q-N$ Nijmegen continuity questionnaire-Norwegian version, $b$ unstandardized estimated regression coefficient, $C I$ confidence interval, Personall NCQ-N personal continuity ("knows me"), Personal 2 NCQ-N personal continuity ("shows commitment"), Team NCQ-N team continuity (within somatic rehabilitation), Crossboundary NCQ-N cross-boundary continuity (between rehabilitation centres and general practitioner in municipality)

${ }^{\text {a}}$ Fully adjusted model is adjusted for: patients' age group, sex, health conditions, education level, marital status and baseline dependent variable subscale score (EQ-VAS)

readmission rates, reduced cost, and increased patient satisfaction as outcomes $[12,16,54,55]$. However, relatively few studies have investigated the associations between continuity of care and patient-rated health outcomes. The present study expands knowledge in this field, revealing a significant association between both personal continuity and team continuity in the rehabilitation team on one hand, and improved health after rehabilitation stay on the other. However, we found no association between continuity of care and changes in the level of disability. These findings indicate a need for more research to verify the impact of continuity of care on patients' outcomes, preferably with more direct measures of health and functioning.

The importance of teams working towards shared goals using a shared approach in health care settings has a wellestablished theoretical and empirical basis, and found to positively influence the quality and continuity of patient care [56-59]. One would therefore assume that a higher score on RC in rehabilitation teams would positively affect patients' health and disability. This present study found that the neoplasms patient group reported a greater improvement in function compared to the other patient groups included. This is in line with previous research that found communication in interprofessional teams to positively impact patient outcomes of cancer care [60]. In our study, this patient group showed the most marked improvement in functioning during the study period. One explanation for this finding could be that this patient group represents a selection of patients who had recently undergone treatment prior to commencing a rehabilitation stay and therefore could be more inclined to be in a phase of recovery where the intervention by rehabilitation teams is especially useful. Patients with nervous system diseases treated by teams with better team functions as measured by RC reported a decrease in health, as measured by EQ-VAS. These patients often have progressive diseases, and one explanation for this finding could be that patients with most serious condition are of greater need for team functions due to a more severe decline in health over time, compared to other diagnosis groups included in this study.

In a previous study, we found that RC communication and relationships in teams were inversely associated with personal continuity as reported by the patient after rehabilitation [18]. Thus, patients treated by a well-functioning team, as defined by RC, were unlikely to specifically have a close relationship with the most important professional during their rehabilitation stay. This is contradictory to previous research reporting that team-based models was associated with increased social participation among stroke patients [61]. However, in these models the patient had a defined coordinator, responsible for systematic follow-up after a rehabilitation process. The present study found an association between personal continuity and improvement in health, as measured by EQ-VAS. This effect of personal continuity is well documented in other care settings $[16,17,21,62]$. Further, in accordance with previous research [12, 55], this current study found continuity of care to positively influence patient-rated changes in health after a rehabilitation stay. One explanation for these findings could be that continuity of care as defined and experienced by patients may differ from continuity of care as defined by health care professionals. The lack of personal continuity might be a limitation of team-based care and should be taken into account when organising rehabilitation care.

Since the present study focused on the health outcomes after rehabilitation, we also looked at cross-boundary continuity between rehabilitation centres and primary health care. Patients may have received health care services in the municipality to follow up interventions received at the rehabilitation centre. Interprofessional rehabilitation teams communicate with other health care professionals across settings, and the current results revealed that better cross-boundary continuity in the NCQ-N was associated with improved health outcomes. This finding is in line with previous studies reporting that a lack of continuity across settings was associated with an increased risk of inactivity, falls and readmission among stroke patients [63]. Further, previous studies have shown that continuity of care after hospital discharge was associated with a reduced risk of death and readmission to hospital $[54,55]$. 


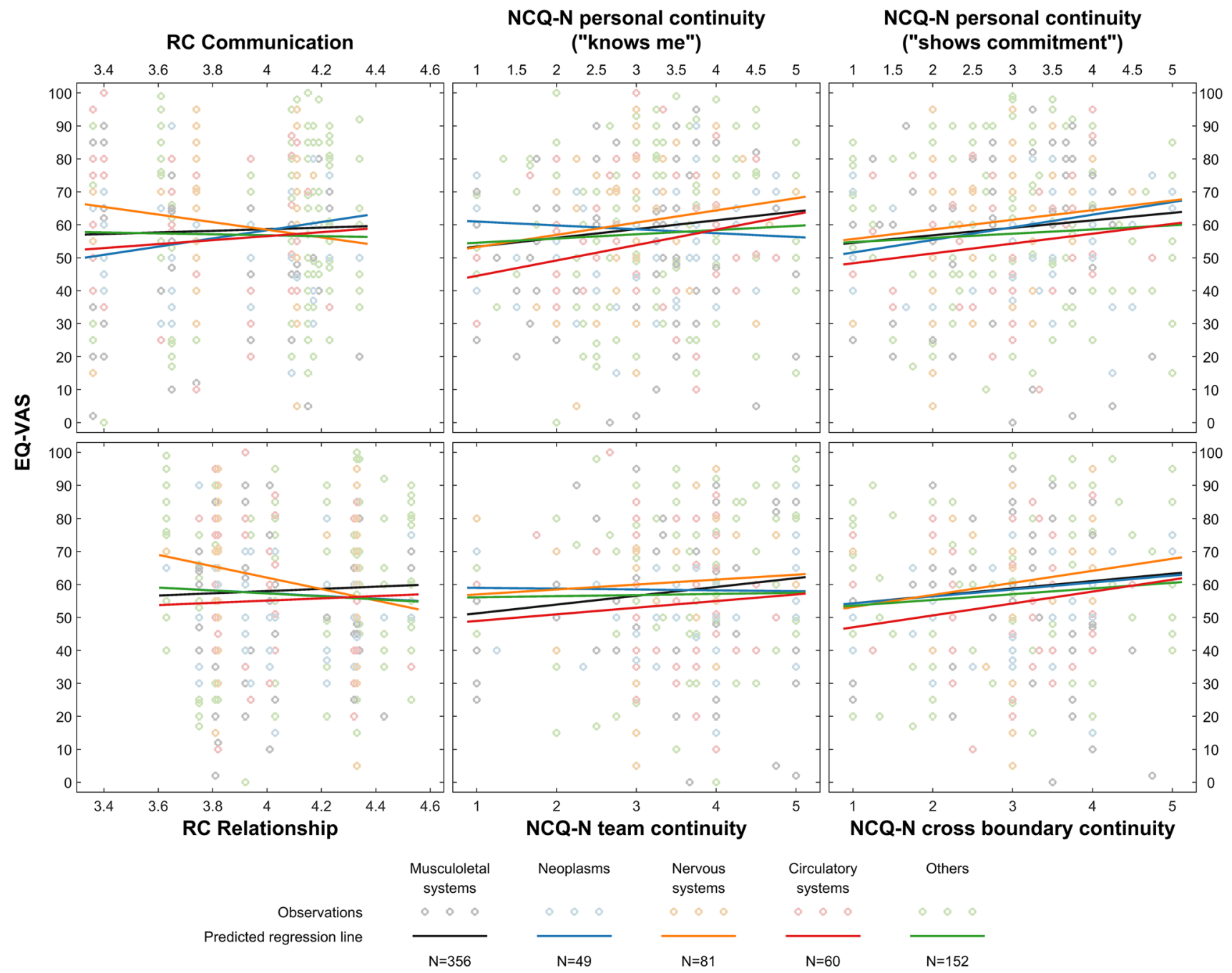

Fig. 3 Associations of relational coordination subscale scores in interprofessional teams and patient-rated continuity of care subscale scores with the EuroQol EQ-VAS health state score with patients

grouped by ICD-10 referral diagnoses $(N=701)$. EQ-VAS EuroQol EQ-VAS, $R C$ relational coordination, $N C Q-N$ Nijmegen continuity questionnaire-Norwegian version

\section{Study strengths and limitations}

An important strength of the current study was the longitudinal design and the comprehensive study population with a broad range of health conditions. In addition, this study included patients who were accepted for somatic rehabilitation in all rehabilitation centres in a defined geographical area (Western Norway), combined with data collection from employees working in interprofessional rehabilitation teams. However, a major limitation was the low response rate at baseline (34\%) and at 1-year follow-up (25\% of the patients recruited at baseline), which may have resulted in selection bias and problems regarding representability. A further limitation was loss of participants at 1-year followup. As non-responders at follow-up seemed to be younger and more often male compared to the responders, an attrition bias could have affected findings. Changes in health at 1-year follow-up could be smaller due to including a sample with a higher mean age and increased number of women.

Strength of the current study was the use of validated generic survey instruments, which enabled us to study a heterogeneous rehabilitation patient cohort. The instruments have shown satisfactory psychometric properties in terms of factor structure and reliability, and the WHODAS 2.0 had satisfactory test-retest reliability [22]. The instruments used were valid and reliable for capturing patient-rated health and disability. However, several limitations regarding the included instruments should be considered. The NCQ-N included the response option "don't know", which, in this study, was set as "missing". This resulted in a relatively large number of missing data points. However, using a flexible multiple imputation method for handling missing data 
reduced the potential effects of bias due to a large number of missing data points in the NCQ-N responses. The low variance in RC between teams may make it difficult to disclose eventual associations between RC in teams and patient-rated outcomes, and our findings should be interpreted with this precaution. The results of the analyses regarding referral diagnosis groups should be interpreted cautiously as some patient groups were relatively small and our findings may therefore not be generalizable to these groups at large. A further potential limitation is that patients in the present study reported mild to moderate disability level according to the WHODAS 2.0 global scale, which may limit the generalisability of the current results to populations with more severe disability.

\section{Conclusion}

The current study revealed that better personal, team and cross-boundary continuity of rehabilitation care was associated with improved health after rehabilitation. Measures of patient-rated personal, team and cross-boundary continuity may be a promising indicator of the quality of rehabilitation care. However, our findings did not reveal any associations between RC in interprofessional teams and self-rated health or disability among rehabilitation patients. More research is needed to understand the effects of team functioning in interprofessional rehabilitation teams on patient health outcomes.

Acknowledgements We thank all personnel at all rehabilitation centres in Western Norway for their participation and recruiting patients for this study. We thank Benjamin Knight, M.Sc., from Edanz Group (http://www.edanzediting.com/ac) for editing a draft of this manuscript.

Authors' contribution $\mathrm{MH}, \varnothing \mathrm{H}$ and $\mathrm{SG}$ developed the project idea and contributed to writing the paper. MH, VPM and JA performed the statistical analyses and interpreted the results. VPM and EB contributed to writing the paper. All authors have approved the final version.

Funding The main author is employed at Haukeland University Hospital, and the study is performed through her position as a Ph.D. candidate.

Availability of data and materials The raw data are property of the research unit in the Bergen Health Authority (Helse Bergen) and are available on a reasonable request.

\section{Compliance with ethical standards}

Conflict of interest The authors declare that they have no conflict of interest.

Consent for publication Not applicable.

Ethical approval The study was approved by the Regional Ethics Committee West in Norway (REK-No. 2014-1636).
All procedures were in accordance with the ethical standards of the Regional research committee and with the Declaration of Helsinki 1964 and it later amendments.

Informed consent Informed consent was obtained from all individual participant included in the study, also accepting the linkage to register data.

Open Access This article is distributed under the terms of the Creative Commons Attribution 4.0 International License (http://creativeco mmons.org/licenses/by/4.0/), which permits unrestricted use, distribution, and reproduction in any medium, provided you give appropriate credit to the original author(s) and the source, provide a link to the Creative Commons license, and indicate if changes were made.

\section{References}

1. European Physical and Rehabilitation Medicine Bodies Alliance. (2018). White book on physical and rehabilitation medicine in Europe. Introductions, executive summary, and methodology. Eur J Phys Rehabil Med, 54(2), 125-155. https://doi.org/10.23736/ s1973-9087.18.05143-2.

2. European Physical and Rehabilitation Medicine Bodies Alliance. (2018). White Book on Physical and Rehabilitation Medicine (PRM) in Europe. Chapter 1. Definitions and concepts of PRM. Eur J Phys Rehabil Med, 54(2), 156-165. https://doi.org/10.23736 /s1973-9087.18.05144-4.

3. Harty, M., Griesel, M., \& van der Merwe, A. (2011). The ICF as a common language for rehabilitation goal-setting: Comparing client and professional priorities. Health Qual Life Outcomes, 9, 87. https://doi.org/10.1186/1477-7525-9-87.

4. Giuliano, C., Parmenter, B. J., Baker, M. K., Mitchell, B. L., Williams, A. D., Lyndon, K., et al. (2017). Cardiac rehabilitation for patients with coronary artery disease: A practical guide to enhance patient outcomes through continuity of care. Clin Med Insights Cardiol, 11, 1179546817710028. https://doi.org/10.1177/11795 46817710028.

5. Donabedian, A. (1988). The quality of care. How can it be assessed? JAMA, 260(12), 1743-1748.

6. Sinclair, L. B., Lingard, L. A., \& Mohabeer, R. N. (2009). What's so great about rehabilitation teams? An ethnographic study of interprofessional collaboration in a rehabilitation unit. Archives of Physical Medicine and Rehabilitation, 90(7), 1196-1201. https ://doi.org/10.1016/j.apmr.2009.01.021.

7. Momsen, A. M., Rasmussen, J. O., Nielsen, C. V., Iversen, M. D., \& Lund, H. (2012). Multidisciplinary team care in rehabilitation: An overview of reviews. Journal of Rehabilitation Medicine, 44(11), 901-912. https://doi.org/10.2340/16501977-1040.

8. Reeves, S., Pelone, F., Harrison, R., Goldman, J., \& Zwarenstein, M. (2017). Interprofessional collaboration to improve professional practice and healthcare outcomes. Cochrane Database Syst Rev. https://doi.org/10.1002/14651858.cd000072.pub3.

9. Gittell, J. H., Fairfield, K. M., Bierbaum, B., Head, W., Jackson, R., Kelly, M., et al. (2000). Impact of relational coordination on quality of care, postoperative pain and functioning, and length of stay: A nine-hospital study of surgical patients. Medical Care, 38(8), 807-819.

10. Ghaffari, A., Wells, R., Creel, L., \& Sianez, M. (2018). A relational perspective on care coordination. Health Care Manage Review. https://doi.org/10.1097/hmr.0000000000000208.

11. Reid, R. J., McKendry, R., \& Haggerty, J. (2002). Defusing the Confusion: Concepts and Measures of Continuity of Health 
Care: Final Report: Canadian Health Services Research Foundation $=$ Fondation canadienne de la recherche sur les Services de santé.

12. van Walraven, C., Oake, N., Jennings, A., \& Forster, A. J. (2010). The association between continuity of care and outcomes: A systematic and critical review. Journal of Evaluation in Clinical Practice, 16(5), 947-956. https://doi.org/10.111 $1 / \mathrm{j} .1365-2753.2009 .01235 . x$.

13. Haggerty, J. L., Reid, R. J., Freeman, G. K., Starfield, B. H., Adair, C. E., \& McKendry, R. (2003). Continuity of care: A multidisciplinary review. BMJ, 327(7425), 1219-1221. https:// doi.org/10.1136/bmj.327.7425.1219.

14. Uijen, A. A., Bischoff, E. W., Schellevis, F. G., Bor, H. H., van den Bosch, W. J., \& Schers, H. J. (2012). Continuity in different care modes and its relationship to quality of life: A randomised controlled trial in patients with COPD. British Journal of General Practice, 62(599), 422-428. https://doi.org/10.3399/bjgp12X649 115.

15. Uijen, A. A., Schers, H. J., \& van Weel, C. (2010). Continuity of care preferably measured from the patients' perspective. Journal of Clinical Epidemiology, 63(9), 998-999. https://doi. org/10.1016/j.jclinepi.2010.03.015.

16. Pandhi, N., \& Saultz, J. W. (2006). Patients' perceptions of interpersonal continuity of care. Journal of the American Board of Family Medicine, 19(4), 390-397.

17. Saultz, J. W., \& Albedaiwi, W. (2004). Interpersonal continuity of care and patient satisfaction: A critical review. Annals of Family Medicine, 2(5), 445-451.

18. Hustoft, M., Biringer, E., Gjesdal, S., Assmus, J., \& Hetlevik, O. (2018). Relational coordination in interprofessional teams and its effect on patient-reported benefit and continuity of care: A prospective cohort study from rehabilitation centres in Western Norway. BMC Health Services Research, 18(1), 719. https://doi. org/10.1186/s12913-018-3536-5.

19. Uijen, A. A., Schers, H. J., Schene, A. H., Schellevis, F. G., Lucassen, P., \& van den Bosch, W. J. (2014). Experienced continuity of care in patients at risk for depression in primary care. The European Journal of General Practice, 20(3), 161-166. https:// doi.org/10.3109/13814788.2013.828201.

20. Lee, C. T., Doran, D. M., Tourangeau, A. E., \& Fleshner, N. E. (2014). Perceived quality of interprofessional interactions between physicians and nurses in oncology outpatient clinics. European journal of oncology nursing : The official journal of European Oncology Nursing Society, 18(6), 619-625. https://doi. org/10.1016/j.ejon.2014.06.004.

21. Medina-Mirapeix, F., Oliveira-Sousa, S. L., Sobral-Ferreira, M., Montilla-Herrador, J., Jimeno-Serrano, F. J., \& Escolar-Reina, P. (2013). What elements of the informational, management, and relational continuity are associated with patient satisfaction with rehabilitation care and global rating change? Archives of Physical Medicine and Rehabilitation, 94(11), 2248-2254. https://doi. org/10.1016/j.apmr.2013.04.018.

22. Moen, V. P., Drageset, J., Eide, G. E., Klokkerud, M., \& Gjesdal, S. (2017). Validation of World Health Organization Assessment Schedule 2.0 in specialized somatic rehabilitation services in Norway. Quality of Life Research, 26(2), 505-514. https://doi. org/10.1007/s11136-016-1384-5

23. Moen, V. P., Drageset, J., Eide, G. E., \& Gjesdal, S. (2018). Dimensions and predictors of disability-A baseline study of patients entering somatic rehabilitation in secondary care. PLOS ONE, 13(3), e0193761. https://doi.org/10.1371/journ al.pone.0193761.

24. Garin, O., Ayuso-Mateos, J. L., Almansa, J., Nieto, M., Chatterji, S., Vilagut, G., et al. (2010). Validation of the "World Health Organization Disability Assessment Schedule, WHODAS-2" in patients with chronic diseases. Health and Quality of Life Outcomes, 8(1), 51. https://doi.org/10.1186/1477-7525-8-51.

25. Posl, M., Cieza, A., \& Stucki, G. (2007). Psychometric properties of the WHODASII in rehabilitation patients. Quality of Life Research, 16, 1. https://doi.org/10.1007/s11136-007-9259-4.

26. Ustun, T., Kostanjsek, N., Chatterji, S., \& Rehm, J. (2010). Measuring health and disability: Manual for WHO Disability Assessment Schedule (WHODAS 2.0). Geneva: World Health Organization.

27. Cieza, A., \& Stucki, G. (2005). Content comparison of healthrelated quality of life (HRQOL) instruments based on the international classification of functioning, disability and health (ICF). Quality of Life Research, 14(5), 1225-1237.

28. Federici, S., Bracalenti, M., Meloni, F., \& Luciano, J. V. (2017). World Health Organization disability assessment schedule 2.0: An international systematic review. Disability and Rehabilitation, 39(23), 2347-2380. https://doi.org/10.1080/09638 288.2016.1223177.

29. Schlote, A., Richter, M., Wunderlich, M. T., Poppendick, U., Moller, C., Schwelm, K., et al. (2009). WHODAS II with people after stroke and their relatives. Disability and Rehabilitation, 31(11), 855-864. https://doi.org/10.1080/09638280802355262.

30. World Health Organization. (2018). WHO Disability Assessment Schedule 2.0 (WHODAS 2.0). http://www.who.int/classifications /icf/whodasii/en/. Accessed 15 May 2019.

31. Nolan, C. M., Longworth, L., Lord, J., Canavan, J. L., Jones, S. E., Kon, S. S., et al. (2016). The EQ-5D-5L health status questionnaire in COPD: Validity, responsiveness and minimum important difference. Thorax, 71(6), 493-500. https://doi.org/10.1136/thora xjnl-2015-207782.

32. Chen, P., Lin, K. C., Liing, R. J., Wu, C. Y., Chen, C. L., \& Chang, K. C. (2016). Validity, responsiveness, and minimal clinically important difference of EQ-5D-5L in stroke patients undergoing rehabilitation. Quality of Life Research, 25(6), 1585-1596. https ://doi.org/10.1007/s11136-015-1196-z.

33. Feng, Y., Parkin, D., \& Devlin, N. J. (2014). Assessing the performance of the EQ-VAS in the NHS PROMs programme. Quality of Life Research, 23(3), 977-989. https://doi.org/10.1007/s1113 6-013-0537-z.

34. van Reenen., \& Janssen, B. (2015). EQ-5D-5L user guide. Basic information on how to use the EQ-5D-5L instrument. (Vol. 2.1). Rotterdam, Nederland.

35. Brazier, J., Jones, N., \& Kind, P. (1993). Testing the validity of the Euroqol and comparing it with the SF-36 health survey questionnaire. Quality of Life Research, 2(3), 169-180.

36. Linde, L., Sorensen, J., Ostergaard, M., Horslev-Petersen, K., \& Hetland, M. L. (2008). Health-related quality of life: Validity, reliability, and responsiveness of SF-36, 15D, EQ-5D [corrected] RAQoL, and HAQ in patients with rheumatoid arthritis. Journal of Rheumatology, 35(8), 1528-1537.

37. Obradovic, M., Lal, A., \& Liedgens, H. (2013). Validity and responsiveness of EuroQol-5 dimension (EQ-5D) versus Short Form-6 dimension (SF-6D) questionnaire in chronic pain. Health Qualaity Life Outcomes, 11, 110. https://doi. org/10.1186/1477-7525-11-110.

38. Ryan, T., Enderby, P., \& Rigby, A. S. (2006). A randomized controlled trial to evaluate intensity of community-based rehabilitation provision following stroke or hip fracture in old age. Clin Rehabil, 20(2), 123-131.

39. Heise, M., Muller, M., Fischer, U., \& Grill, E. (2016). Quality of life in older individuals with joint contractures in geriatric care settings. Quality of Life Research, 25(9), 2269-2281. https://doi. org/10.1007/s11136-016-1262-1.

40. Parker, S. G., Oliver, P., Pennington, M., Bond, J., Jagger, C., Enderby, P., et al. (2011). Rehabilitation of older patients: Day hospital compared with rehabilitation at home, clinical outcomes. 
Age Ageing, 40(5), 557-562. https://doi.org/10.1093/ageing/afr04 6.

41. Valentine, M. A., Nembhard, I. M., \& Edmondson, A. C. (2015). Measuring teamwork in health care settings: A review of survey instruments. Medical Care, 53(4), e16-e30. https://doi. org/10.1097/MLR.0b013e31827feef6.

42. Hustoft, M., Hetlevik, O., Assmus, J., Storkson, S., Gjesdal, S., \& Biringer, E. (2018). Communication and relational ties in interprofessional teams in Norwegian specialized health care: A multicentre study of relational coordination. International Journal of Integrated Care, 18(2), 9. https://doi.org/10.5334/ijic.3432.

43. Gittell, J. H. (2012). Relational Coordination: Guidelines for Theory, Mearsurement and Analysis. Brandeis: Heller School, Brandeis University USA: Relational Coordination Research Collaboration.

44. Cramm, J. M., Hoeijmakers, M., \& Nieboer, A. P. (2014). Relational coordination between community health nurses and other professionals in delivering care to community-dwelling frail people. Journal of Nurse Management, 22(2), 170-176. https://doi. org/10.1111/jonm.12041.

45. Hartgerink, J. M., Cramm, J. M., Bakker, T. J., van Eijsden, R. A., Mackenbach, J. P., \& Nieboer, A. P. (2014). The importance of relational coordination for integrated care delivery to older patients in the hospital. Journal of Nursing Management, 22(2), 248-256. https://doi.org/10.1111/j.1365-2834.2012.01481.x.

46. Uijen, A. A., Schellevis, F. G., van den Bosch, W. J., Mokkink, H. G., van Weel, C., \& Schers, H. J. (2011). Nijmegen Continuity Questionnaire: Development and testing of a questionnaire that measures continuity of care. Journal of Clinical Epidemiology, 64(12), 1391-1399. https://doi.org/10.1016/j.jclin epi.2011.03.006.

47. Uijen, A. A., Schers, H. J., Schellevis, F. G., Mokkink, H. G., van Weel, C., \& van den Bosch, W. J. (2012). Measuring continuity of care: Psychometric properties of the Nijmegen Continuity Questionnaire. British Journal of General Practice, 62(600), 949-957. https://doi.org/10.3399/bjgp12X652364.

48. Uijen, A. A. (2012). Continuity of Care Perspectives of the patient with chronic illness. Netherland: Radboud University Nijmegen Medical Centre.

49. Hetlevik, O., Hustoft, M., Uijen, A., Assmus, J., \& Gjesdal, S. (2017). Patient perspectives on continuity of care: Adaption and preliminary psychometric assessment of a Norwegian version of the Nijmegen Continuity Questionnaire (NCQ-N). BMC Health Services Research, 17(1), 760. https://doi.org/10.1186/s1291 3-017-2706-1.

50. van Buuren, S. (2018). Flexible imputation of missing data (2nd ed.). New York: CRC Press, Taylor \& Francis Group.

51. Veierød, M., Lydersen, S., \& Laake, P. (2012). Medical statistics in clinical and epidemiological reseach (1st ed.). Oslo/Trondheim: Gyldendal Akademisk.
52. IBM SPSS Statistics for Windows, Version 24.0 NY: IBM Corp.

53. Stata statistical software: Release 15 (2017). TX: StataCorp LP.

54. van Walraven, C., Mamdani, M., Fang, J., \& Austin, P. (2004). Continuity of care and patient outcomes after hospital discharge. Journal of General Internal Medicine, 19, 624-631.

55. Cabana, M. D., \& Jee, S. H. (2004). Does continuity of care improve patient outcomes? Journal of Family Practice, 53(12), 974-980.

56. Havens, D. S., Vasey, J., Gittell, J. H., \& Lin, W. T. (2010). Relational coordination among nurses and other providers: Impact on the quality of patient care. Journal of Nursing Management, 18(8), 926-937. https://doi.org/10.1111/j.1365-2834.2010.01138.x.

57. Gittell, J. H., Godfrey, M., \& Thistlethwaite, J. (2013). Interprofessional collaborative practice and relational coordination: Improving healthcare through relationships. Journal of Interprofessional Care, 27(3), 210-213. https://doi.org/10.3109/13561 820.2012 .730564 .

58. Neumann, V., Gutenbrunner, C., Fialka-Moser, V., Christodoulou, N., Varela, E., Giustini, A., et al. (2010). Interdisciplinary team working in physical and rehabilitation medicine. Journal of Rehabilitation Medicine, 42(1), 4-8. https://doi.org/10.2340/16501 977-0483.

59. Yeager, S. (2005). Interdisciplinary collaboration: The heart and soul of health care. Critical Care Nursing Clinics of North America, 17(2), 143-148. https://doi.org/10.1016/j.ccell.2005.01.003.

60. Tremblay, D., Roberge, D., Touati, N., Maunsell, E., \& Berbiche, D. (2017). Effects of interdisciplinary teamwork on patientreported experience of cancer care. BMC Health Services Res, 17(1), 218. https://doi.org/10.1186/s12913-017-2166-7.

61. Fens, M., van Heugten, C. M., Beusmans, G., Metsemakers, J., Kester, A., \& Limburg, M. (2014). Effect of a stroke-specific follow-up care model on the quality of life of stroke patients and caregivers: A controlled trial. Journal of Rehabilitation Medicine, 46(1), 7-15. https://doi.org/10.2340/16501977-1239.

62. Mendes, F. R., Gemito, M. L., Caldeira, E. D., Serra, I. D., \& Casas-Novas, M. V. (2017). Continuity of care from the perspective of users. Cien Saude Colet, 22(3), 841-853. https://doi. org/10.1590/1413-81232017223.26292015.

63. Freburger, J. K., Li, D., Johnson, A. M., \& Fraher, E. P. (2018). Physical and occupational therapy from the acute to community setting after stroke: Predictors of use, continuity of care, and timeliness of care. Archives of Physical Medicine and Rehabilitation, 99(6), 1077-1089.e1077. https://doi.org/10.1016/j. apmr.2017.03.007.

Publisher's Note Springer Nature remains neutral with regard to jurisdictional claims in published maps and institutional affiliations. 